ENCYCLOPEDDIE Encyclopédie berbère

BERBERE

$7 \mid 1989$

7 | Asarakae - Aurès

\title{
Augustin (saint)
}

\section{S. Lancel}

\section{OpenEdition}

Journals

Édition électronique

URL : http://journals.openedition.org/encyclopedieberbere/1222

DOI : 10.4000/encyclopedieberbere.1222

ISSN : 2262-7197

\section{Éditeur}

Peeters Publishers

\section{Édition imprimée}

Date de publication : 1 janvier 1989

Pagination : 1055-1065

ISBN : 2-85744-443-5

ISSN : 1015-7344

\section{Référence électronique}

S. Lancel, «Augustin (saint) », Encyclopédie berbère [En ligne], 7 | 1989, document A319, mis en ligne le 01 décembre 2012, consulté le 18 octobre 2020. URL : http://journals.openedition.org/ encyclopedieberbere/1222 ; DOI : https://doi.org/10.4000/encyclopedieberbere.1222

Ce document a été généré automatiquement le 18 octobre 2020

(C) Tous droits réservés 


\section{Augustin (saint)}

\section{S. Lancel}

1 Saint Augustin appartient à la culture universelle, et les diverses composantes de son expérience d'homme, de pasteur, d'écrivain, de théologien, de mystique sont naturellement indissociables les unes des autres. Mais, pour cette présentation d'un grand Africain dans le cadre de cette "Encyclopédie berbère ", on a, tout naturellement aussi, privilégié les aspects proprement africains de cette vie, de cette «carrière ", de cette action.

2 "Berbère ", c'est-à-dire de sang "indigène ", pour l'essentiel, Augustin avait, statistiquement, de fortes chances de l'être, comme la très grande majorité des Romano-Africains de son temps. Passer de cette forte probabilité statistique à l'affirmation d'un statut individuel (cf. par exemple le livre de René Pottier, Saint Augustin le Berbère, Paris, 1945) relève de la fiction romanesque et tendrait à créditer l'évêque d'Hippone d'un parti pris d'africanité nationaliste ou régionaliste dont il a toujours, nous le verrons, été très éloigné. On notera toutefois que si le nom personnel, ou cognomen, de son père, Patricius, appartient à l'onomastique latine la plus banale sous le Bas-Empire, celui de sa mère, Monnica (« Monique ») particulièrement fréquent dans la Numidie d'Hippone, est le diminutif de Monna, nom indigène lui-même bien attesté qui est aussi celui d'une divinité dont le culte est mentionné sur une inscription de Tignica (Aïn Tounga, dans la vallée de la Medjerda). Quant au nom de famille, ou " gentilice ", Aurelius, il pourrait suggérer que leurs ancêtres avaient été naturalisés Romains, avec toute la masse des provinciaux, par le fameux édit de Caracalla, en 212, si du moins les duo nomina, Aurelius Augustinus, ne sont pas, comme on le pense parfois, le fruit d'une confusion née de la répétition de cette séquence dans les listes épiscopales, où le nom d'Aurélius, évêque de Carthage, précède habituellement celui d'Augustinus. Ce dernier nom personnel enfin, si rare - et si ambitieux : littéralement, « le petit Auguste », ou le " petit Empereur » -, les parents l'avaient-ils donné à celui qui devait l'illustrer par prescience d'un destin unique ? Sous ce diminutif allait grandir un enfant dont la gloire posthume, un jour, éclipserait celle des maitres du monde. 


\section{L'enfance et l'éducation ; le milieu familial et social}

3 Augustin est né aux Ides de novembre (le 13 novembre) de l'année 354 à Thagaste, aujourd'hui Souk-Ahras, aux confins algéro-tunisiens, dans cette région montagneuse et boisée du haut cours du Bagrada (actuelle Medjerda) qui faisait traditionnellement partie du pays numide, mais était administrativement rattachée à la province d'Afrique Proconsulaire et se trouvait donc sous la juridiction du proconsul en résidence à Carthage. Le père d'Augustin, Patricius, était un petit propriétaire foncier qui appartenait à la classe des curiales, petite bourgeoisie municipale de plus en plus écrasée sous le poids de sa responsabilité collective en matière d'impôts. En dépit de la modestie de ses ressources, Patricius tint à assurer à son fils l'éducation libérale qui était pour les gens de sa classe le seul passeport pour une meilleure réussite sociale, d'abord, semble-t-il, à Thagaste même, puis, pour les études de grammaire et de rhétorique, à une trentaine de kilomètres au sud de Thagaste, à Madaure, dont une autre gloire locale, Apulée, avait rendu célèbres les écoles de grammatici. Augustin a raconté dans les Confessions son peu de goût, alors, pour l'étude (Conf., I, XII, 19), et plus précisément son aversion pour le grec, qu'il lui fallait apprendre comme une langue totalement étrangère (Conf., I, XIII, 20 ; XIV, 23), alors que pour le latin, qu'il avait appris «au milieu des caresses de ses nourrices " («inter blandimenta nutricum »: Conf., I, XVI, 23), il ne manifestait aucune répugnance et montrait même une grande facilité dans la composition latine (Conf., I, XVII, 27).

En sa seizième année, l'impécuniosité des siens le contraignit à quitter Madaure et à interrompre ses études (Conf., II, III, 5 et 6). Ce fut, à Thagaste, une année de désœuvrement, marquée par les premiers émois de la chair, employée à des jeux défendus, à des maraudes, comme ce vol de poires en un verger voisin de chez lui, analysé plus tard dans les Confessions avec beaucoup de pénétration, mais aussi avec une sévérité qui peut nous paraître maintenant un peu disproportionnée (Conf., II, IV, 9-IX, 17).

\section{Les années de formation et l'expérience carthaginoise}

Grâce à la générosité d'un riche notable de Thagaste, ami de sa famille, Romanianus, Augustin put aller continuer ses études de rhétorique à Carthage, avec l'aide également des subsides de sa mère, lorsque son père mourut, peu après son départ (Conf., III, IV, 7). Sur ce jeune homme sensible et si doué, la grande métropole africaine fit forte impression. Capitale de la débauche, c'est ainsi que la ville lui apparût d'abord: "J'arrivai à Carthage et tout autour de moi bouillonnait la chaudière des honteuses amours » (Conf., III, I, 1 ou Carthago-sartago fait un jeu de mot intraduisible). Il ne tarda pas à succomber à ces plaisirs : plaisirs de la chair, mais aussi plaisir du théâtre, que le jeune homme découvrit avec ravissement (Conf., III, II, 2). Parallèlement, il se révélait excellent étudiant, répugnant aux "chahuts " auxquels se livraient certains de ses camarades, les eversores (Conf., III, III, 6), se liant d'amitié avec des condisciples provinciaux comme lui que les hasards de la vie lui feront retrouver plus tard, comme Vincentius, qui deviendra évêque de l'Église donatiste à Cartennae (Ténès) (Ep. 93, 51). Son premier émoi intellectuel lui fut procuré par la lecture de l'Hortensius de Cicéron; il avait alors dix-neuf ans et voyait s'ouvrir devant lui le monde de la pensée (Conf., III, IV, 7-8). Alors, le souvenir d'une première et fugitive imprégnation chrétienne, qu'il devait 
à sa mère en son enfance (Conf., I, XI, 17), lui suggéra de lire, à la suite de l'Hortensius, les Écritures : mais il ne put entrer dans la Bible, dont le style le rebuta (Conf., III, V, 9).

6 A la même époque, un peu avant sa vingtième année (on était en 374), il se laissa séduire par les idées des Manichéens et devint lui-même, pendant neuf ans, un « auditeur » de la secte (Conf., III, XI, 20 ; IV, I, 1). Cette phase manichéenne coïncida avec ses débuts dans l'enseignement, d'abord chez lui, à Thagaste. Mais la mort d'un ami d'enfance qu'il y avait retrouvé, mort qui l'affecta profondément, le décida à retourner à Carthage (Conf., IV, IV, 7-VI, 11). Dans la capitale, son enseignement de la rhétorique fut un succès: Augustin remporta des concours à plusieurs reprises et fut notamment couronné par le proconsul Helvius Vindicianus, à qui il dut aussi de se détourner de l'astrologie, pour laquelle il avait eu des curiosités (Conf., IV, III, 5). Du groupe de ses étudiants, à Carthage, se détache la figure d'Alypius, originaire comme lui de Thagaste, et qui devait plus tard (en 395) devenir évêque de cette cité. Augustin vivait alors avec une femme dont le nom n'est jamais prononcé, de qui il eut un fils, son unique enfant, Adeodatus, et qui devait rester près de lui pendant près de quinze ans (Conf., IV, II, 2 ; VI, XV, 25).

7 A l'âge de vingt-six ou vingt-sept ans (Conf., IV, XIII, 20-XV, 27), donc vers 380, il composa un premier ouvrage, Le Beau et le Convenable (de pulchro et apto), largement inspiré par la métaphysique manichéenne, qui ne nous est pas parvenu. Cependant, et de façon paradoxale, c'est la recontre en 383 , du «pape » du manichéisme africain, Faustus de Milev, venu donner un enseignement à Carthage, qui va le plus contribuer à commencer à détacher Augustin du manichéisme, Faustus se révélant incapable de lui donner des réponses satisfaisantes aux questions qu'il se pose sur la doctrine (Conf., V, VI, 10-11). A la même époque, las de supporter l'indiscipline et les mauvaises manières des étudiants carthaginois, il décida d'aller s'établir à Rome.

\section{Les années outre-mer et la conversion}

8 On nous permettra de passer un peu rapidement sur ces années italiennes, dont il faut cependant dire tout de suite qu'elles eurent une importante capitale dans l'évolution intellectuelle, morale et spirituelle d'Augustin.

9 Augustin ne s'attarda pas longtemps à Rome où, à peine arrivé, il était tombé malade et avait failli mourir, où, surtout, il connut des désillusions avec ses étudiants, certes moins indisciplinés qu'à Carthage, mais mauvais payeurs (Conf., V, XII, 22). Il brigua et obtint de Symmaque une chaire de rhétorique à Milan et se présenta aussitôt à l'évêque de cette ville, Ambroise, dont la personnalité le séduisit fort et dont l'enseignement ébranla ses convictions manichéennes déjà vacillantes.

10 Sa mère, Monique, l'avait rejoint à Milan et avait arrangé pour son fils un mariage, dans la perspective duquel ce dernier avait renvoyé en Afrique sa concubine, la mère d'Adeodatus. Mais, en fait, Augustin était déjà entré dans une sorte de gestation spirituelle. Rejoint à Milan, en 384, par Alypius, l'ami de toujours, et par Nebridius, un autre intime, Augustin se pose des questions sur la vanité de ses ambitions temporelles et conçoit avec ses deux amis des projets de vie en communauté qui n'aboutissent pas (Conf., VI, VI, 9 ; XIV, 24). Il se détourne définitivement de l'astrologie et se plonge dans des livres néo-platoniciens, traduits en latin, qui, sur le plan intellectuel, préparent sa conversion (Conf., VII, IX, 13-XX, 26). Ces lectures l'amènent aux Evangiles et aux Epîtres 
de saint Paul. Le récit qui lui est fait de la conversion de Victorinus, célèbre rhéteur romain, fait sur lui forte impression (Conf., VIII, II, 3-IV, 5), et plus encore les récits qu'en compagnie d'Alypius lui fait un de ses compatriotes africains, Ponticianus, lequel leur fait connaître Antoine, le moine égyptien, et leur raconte des conversions exemplaires (Conf., VIII, VI, 14-15).

Les voyages de saint Augustin (carte dressée par S. Lancel, dessin de Y. Assié).

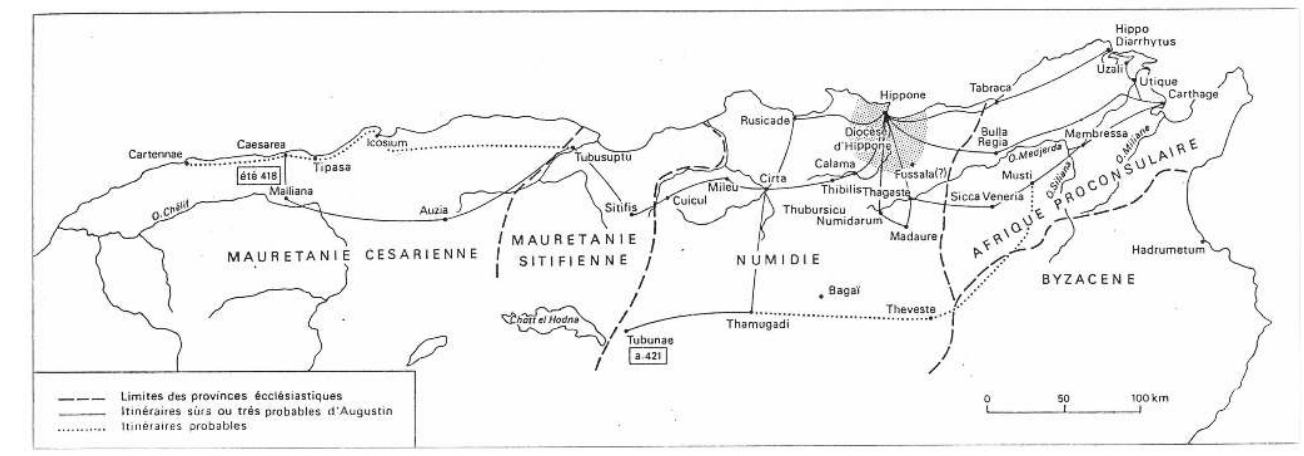

11 La crise décisive survient alors, et se dénoue dans le petit jardin attenant au logis d'Augustin et Alypius à Milan. Entendant, venue de la maison voisine, une voix d'enfant qui disait: "Prends! Lis!", Augustin interprète comme un oracle ce qui était sans doute une comptine ou un refrain et, s'emparant des Écritures qui étaient à portée de main, il y lit un verset de saint Paul (Rom., 13, 13), qui emporte son adhésion, ainsi que celle d'Alypius. Ils décident l'un et l'autre sur le champ de renoncer au monde et de vivre une vie de continence consacrée à Dieu. C'était en août 386, Augustin allait avoir trente-deux ans.

L'automne et l'hiver qui suivirent furent passés à Cassiciacum non loin de Milan, dans la propriété d'un ami. Augustin avait démissionné de sa chaire de rhéteur; il était maintenant catéchumène et étudiait les Psaumes en compagnie de sa mère Monique et d'Alypius (Conf., IX, IV, 7-12). Il mit aussi sa nouvelle liberté à profit pour écrire les premiers Dialogues (le contra Academicos, le de uita beata, le de ordine, les Soliloquia). A Pâques 387, il reçut le baptême des mains d'Ambroise, à Milan, en même temps qu'Alypius et que son fils Adéodat, qui était alors âgé de quatorze ans (Conf., IX, VI, 14). Le séjour italien touchait à sa fin; à l'automne 387 , alors que le petit groupe était à Ostie, sur le chemin du retour, Monique mourut. Augustin et ses amis passèrent l'hiver à Rome (Conf., IX, VIII, 29). L'année suivante, en 388, ils rentrèrent tous en Afrique.

\section{Le Docteur de l'Église et l'évêque africain}

13 A l'automne 388, Augustin prit terre à Carthage mais ne s'y attarda pas. Il rentra à Thagaste, sa ville natale, bien décidé à s'y fixer et à y vivre cette vie en communauté dont les mois passés à Cassiciacum avaient fourni une expérience préparatoire; et, de fait, pendant près de trois ans, en compagnie d'Alypius et de quelques amis, avec lesquels il forma une communauté monastique, il vécut une vie cénobitique dans la maison paternelle et sur ses terres, à la propriété desquelles il avait renoncé (Possidius, Vita Aug., III, 1-2). Peu après son retour à Thagaste, la mort prématurée de son fils Adéodat avait coupé son dernier lien charnel avec son passé (Conf., IX, VI, 14). Il 
composa plusieurs ouvrages durant cette période: le de magistro, le de Genesi contra Manicheos, le de uera religione, et termina le de musica commencé en 387 à Milan.

Le destin l'avait cependant réservé à d'autres fins que cet otium chrétien. Un jour de 391, comme il se trouvait à Hippone (Hippo Regius, aujourd'hui Annaba) alors que le vieil évêque de la ville, Valerius, avait fait état devant ses fidèles de l'impérieuse nécessité pour leur église de pourvoir à l'ordination d'un prêtre, ceux-ci s'emparèrent de lui et lui imposèrent la charge presbytérale (Possidius, Vita Aug., IV). Ordonné prêtre, Augustin reçut bientôt de son évêque mission de prêcher en chaire, fait jusque là sans précédent en Afrique, et c'est ainsi que, lors du concile général qui, en 393, réunit à Hippone tous les évêques africains, Augustin fut chargé de faire l'exposé doctrinal Sur la foi et le symbole (de fide et symbolo). Peu après, pour préparer sa succession, Valerius décidait de faire d'Augustin son " évêque-coadjuteur » (Possidius, Vita Aug., VIII, 2, 15).

Augustin succéda à Valerius en 395 : une « carrière » épiscopale de plus de trente cinq années s'ouvrait devant lui, au cours desquelles l'évêque d'Hippone fut intimement mêlé, et bien au-delà des limites d'un diocèse parmi les plus vastes, aux réalités de tous ordres - religieuses, ecclésiastiques, sociales, voire même économiques - de la vie des provinces d'Afrique. Dans le cadre de cette notice, c'est à ces «engagements » qu'on s'arrêtera surtout.

\section{La lutte antidonatiste (395-411)}

Parmi ces « engagements » divers, rien peut-être ne confronta plus Augustin avec les réalités africaines, non seulement religieuses, ou ecclésiastiques, mais aussi sociales, au sens le plus large du terme, que son engagement personnel dans la lutte antidonatiste. Lorsqu'il devint évêque d'Hippone, la puissance de la secte était à son apogée. En 394, le concile schismatique de Bagaï, en Numidie, avait pu réunir trois cent dix évêques, et l'on était alors dans les années terribles de ce qu'Augustin a appelé le "gémissement de l'Afrique ", lorsque Optat de Timgad, l'« êvêque-brigand ", régnait sans partage sur la Numidie avec ses bandes de circoncellions, et s'apprêtait à faire alliance avec le comte Gildon dans sa tentative d'usurpation du pouvoir impérial, en 397.

Ce «dossier » du donatisme était le premier dont Augustin, en tant que pasteur, avait dû se saisir. D'abord pour tenter d'engager le dialogue avec ses collègues schismatiques. Il était encore prêtre quand, avant 395, il s'adressait à Maximinus, évêque donatiste du castellum Sinitense, limitrophe d'Hippone, pour l'exhorter à un échange exempt, de part et d'autre, de toute argumentation polémique (Ep. 23, 6). En 395/396, devenu évêque auxiliaire de Valerius, il faisait, sur le même ton, la même ouverture à l'évêque donatiste de sa propre ville épiscopale, Proculianus (Ep. 33, 4 et 5). Ses déboires avec ce dernier ne découragèrent pas Augustin de poursuivre ses tentatives d'échanges et de rencontres. C'est ainsi qu'au courant de l'été 395, encore évêque auxiliaire, il saisit l'occasion de son passage à Thubursicu Numidarum (Khamissa) pour solliciter un entretien avec l'évêque donatiste du lieu, Fortunius. Le succès de cette rencontre, à l'issue de laquelle on avait même envisagé d'organiser une conférence contradictoire réunissant dix évêques de chaque partie, ne pouvait qu'entretenir Augustin dans sa volonté de dialogue (Ep. 44). Mettant à profit ce qu'on lui avait rapporté de son désir de correspondre, il écrivit ainsi, après 396, à Honoratus, évêque donatiste d'un diocèse inconnu, mais proche d'Hippone (Ep. 52, 1). En 399/400, Augustin s'autorisait sans perdre de temps des bonnes dispositions de son voisin Crispinus, l'évêque schismatique 
de Calama (Guelma) - prêt, selon la rumeur publique, à discuter avec lui - pour l'engager à débattre, mais par lettres, sur les raisons du schisme (Ep. 51, 1). Peu après, Crispinus rebaptisait les colons du fundus Mappaliensis, dont il était propriétaire, et Augustin en prenait occasion pour lui proposer un débat, de vive voix cette fois, dont les colons en question devaient être les arbitres (Ep. 66, 2). Le refus de Crispinus l'obligea à renoncer à ce projet.

18 A défaut de résultats toujours positifs, ces démarches avaient permis à Augustin de commencer à élaborer toute une argumentation ébauchée dès 394 dans le Psalmus contra partent Donati, puis largement développée à partir de 400 dans les grands traités antidonatistes; d'abord dans le contra epistulam Parmeniani, puis, entre 401 et 405, dans le de baptismo et le contra litteras Petiliani, enfin, un peu plus tard, dans le contra Cresconium. Parallèlement, Augustin s'employait, lors des conciles généraux réunis par Aurèlius de Carthage, pour qu'on parvienne à une confrontation entre les deux Églises. En particulier, son influence, sinon sa main, est sensible dans la préparation et la rédaction des mesures prises par le concile du 13 septembre 401, qui donnait mandat à une délégation d'évêques catholiques d'aller discuter avec leurs collègues donatistes (Concilia Africae, éd. Ch. Munier, CCL, 149, p. 199-201). Deux ans plus tard, le concile du 25 août 403 précisait cette démarche, rédigeait une forma conuentionis donatistarum, c'est-à-dire une formule de convocation qui devait être notifiée dans chaque cité à l'évêque schismatique par les soins des magistrats municipaux (Concilia Africae, CCL, 149, p. 210-211). Cette fois-ci encore, l'affaire n'eut pas de suite, mais c'était comme une répétition générale des procédures qui devaient effectivement aboutir en 411.

Il n'est que de consulter les Actes de la Conférence de Carthage en 411, du moins ce qui nous en est parvenu, pour constater l'importance exceptionnelle du rôle que l'évêque d'Hippone joua dans cette grande confrontation des deux épiscopats, en particulier lors de la troisième séance, celle du 8 juin, où son action fut décisive. Au soir de cette journée, grâce à la clairvoyance, à l'énergie, à l'intelligence tactique et à l'habileté oratoire d'Augustin, les donatistes avaient cause perdue. Restait à éliminer totalement le schisme. L'amitié qui liait Augustin à l'arbitre de la Conférence, le haut dignitaire impérial Marcellinus - à qui il dédia la Cité de Dieu -, ses bonnes relations avec le frère de ce dernier, Apringius, proconsul d'Afrique, l'aidèrent à consolider cette victoire. Pour mieux exploiter le succès de son Église, l'évêque d'Hippone se fit propagandiste ; il rédigea un abrégé des Actes, le Breuiculus conlationis lui-même suivi d'une lettre circulaire intitulée Ad donatistas post conlationem. Il fit en sorte que, dans les années qui suivirent la Conférence, une édition des Actes en fût lue en chaire pendant le carême dans les principaux diocèses d'Afrique. Cà et là, des résistances sporadiques maintenaient l'affaire à l'ordre du jour: à Caesarea (Cherchell), en Maurétanie Césarienne, lorsque Augustin y rencontra l'obstiné schismatique Emeritus, muré dans son mutisme, durant l'été 418 ; à Thamugadi (Timgad), en Numidie, lorsque l'évêque Gaudentius, rebelle aux édits d'union, menaçait en 420 de mettre le feu à sa basilique et d'y périr avec le reste de ses fidèles, ce dont Augustin le dissuada en dialoguant avec lui.

\section{Iter faciendo per Africam : conciles, missions, voyages divers}

La majeure partie d'un grand livre sur Les voyages de saint Augustin est consacrée aux déplacements qu'Augustin, devenu évêque d'Hippone, fut amené à faire en Afrique, 
toujours pour quelque ecclesiastica necessitas, pour quelque affaire pressante de l'Église, qui requérait son habileté, ou son savoir, ou son " autorité ", bref, ce que l'on pourrait appeler son charisme. Augustin avait à ces pérégrinations d'autant plus de mérite que nous savons, par ses confidences réitérées, qu'il répugnait à ces déplacements qui l'arrachaient à ses ouailles, aux ouvrages qu'il avait en train, et que, l'âge venant, et sa santé déclinant, il supportait physiquement de plus en plus mal.

Quand on était évêque, on voyageait d'abord pour assister aux conciles provinciaux et généraux. C'était une obligation, à laquelle Augustin n'a failli qu'exceptionnellement : ainsi n'assistera-t-il pas, début mars 420, à un synode provincial tenu apud Mazacos (?), en Numidie, à cause du froid - qu'il redoutait particulièrement vers la fin de sa vie comme il l'écrit à son ami Alypius (Ep. 22*, CSEL, 88, p. 113). En fait, Augustin a participé régulièrement aux grands rendez-vous de l'épiscopat africain, parfois en province - et non loin de chez lui, comme à Milev (Mila), en Numidie, en $402-$, le plus souvent à Carthage, soit au printemps, soit vers la fin de l'été. Il fut l'un des piliers de la riche série des conciles réunis par le primat d'Afrique, Aurelius, de 393 à 427, faisant le plus souvent partie de ces legati qui, passées les séances plénières, demeuraient en petit comité parfois des semaines entières pour élaborer, sur tel point délicat, canons disciplinaires ou lettres synodales : ce fut le cas en 418 et en 419 (Concilia Africae, CCL, 149, p. 227, p. 229-230).

En vérité, si l'on met bout à bout tous les voyages et les séjours, longs ou brefs, hors d'Hippone, on s'aperçoit que sur ses trente-cinq années d'épiscopat l'évêque a, en fait, passé de longues années en dehors de son diocèse. Carthage d'abord a bénéficié de ses absences, cette Carthage où il s'est passé peu d'années qu'il n'ait prononcé sermons et commentaires sur les Psaumes, faisant le tour de ses basiliques et des ses « régions ». Il y a souvent séjourné des étés entiers, depuis la date du concile plénier (en général fin mai ou début juin) jusqu'au début de l'automne. De retour à Hippone, c'était la fièvre des affaires à régler, qui s'étaient accumulées, des correspondances en retard, des ouvrages ou des libelles de circonstance qu'il lui fallait dicter en toute hâte, pour rattraper le temps perdu ; ainsi, à l'automne 419 , rentré chez lui de Carthage après une absence de plusieurs mois, il faisait dans une lettre à Possidius de Calama le compte de l'impressionnante série des lettres et traités qu'il avait dictés en l'espace de quelques semaines - en employant pour des tractatus populares, c'est-à-dire des sermons qu'il envoyait à Carthage, ses nuits du samedi et du dimanche: en tout six mille lignes, comprenant des textes comme le contra Gaudentium, le contra sermonem Arianorum et le livre I du de natura et origine animae (Ep. 23* A, 3, CSEL, 88, p. 122-123.

La carte que l'on peut tenter de dresser des voyages de saint Augustin ne saurait pleinement rendre compte des réalités physiques de ces pérégrinations, encore moins des multiples contacts dont elles furent l'occasion, ne serait-ce que parce que l'évêque d'Hippone a souvent replacé ses pas dans les mêmes traces, dans les axes qu'il a le plus souvent parcourus ; vers le sud, en demeurant dans sa " Numidie d'Hippone », quand il allait à Calama ou à Thagaste, et surtout vers l'est, quand il se rendait à Carthage, soit par la route du littoral, en passant par Thabraca (Tabarka) et Hippo Diarrhytus (Bizerte), puis Uzalis (El Alia), chez son ami Evodius, soit par la grande route de la vallée de la Medjerda, laquelle comportait trop de variantes d'itinéraires pour que nous puissions en décider. moins rebattues. S'il ne semble pas s'être jamais aventuré en Byzacène (ou, si l'on 
préfère, en Tunisie centrale et méridionale), il lui est arrivé de dépasser, vers l'ouest, les vastes horizons numides. Son voyage le plus lointain le conduisit, durant l'été 418 , en Maurétanie Césarienne (c'est-à-dire dans l'Algérois et l'Oranie actuels) où, sur mandat du pape Zozime, il accomplit une mission d'« inspection ecclésiastique » dont les objectifs ne nous apparaissent que partiellement. Le temps fort et l'étape la plus importante de cette tournée se situèrent dans la capitale provinciale, à Caesarea (Cherchell), où Augustin eut notamment - mais ce n'était pas le but du voyage l'occasion de rencontrer son vieil adversaire Emeritus (Sermo ad Caesariensis ecclesiae plebem, Gesta cum Emerito, CSEL, 53, p.165-196). Presque deux ans plus tard, au printemps de l'année 420 , la délégation reçue de Zozime paraissait toujours valide sous le pontificat de Boniface, et l'arbitrage qu'Augustin s'efforce de rendre dans les difficultés que vivait la communauté de Cartennae (Ténès) suggère que la Maurétanie Césarienne était alors toujours placée sous sa tutelle (Ep. 22* $5-11 ; 23^{*} ; 23^{*} \mathrm{~A}, 5-6=$ CSEL, 88, p. 115-125). Un peu plus tard, vraisemblablement en 421, et en compagnie d'Alypius, l'évêque d'Hippone, traversant toute la Numidie jusqu'à ses confins sud avec la Maurétanie Sitifienne, fit une incursion en territoire militaire, dans une de ces zones du limes, de frontière fortifiée et surveillée, par où transitaient, dûment filtrés par les garnisons romaines, les barbares païens venus du pré-désert (voir, par exemple, pour la zone des Arzuges, dans la région du Chott Djerid, les lettres 46 et 47 échangées entre l'évêque d'Hippone et Publicola). Celui qu'Augustin vint alors rencontrer à Tubunae (Tobna), non loin du Chott el Hodna, allait devenir « comte d'Afrique », et le principal chef militaire de son temps ; mais Bonifatius n'était encore que «tribun ", commandant un secteur clef de ce limes d'Afrique, très lié avec Augustin, avec lequel il était entré en relations dès son arrivée en Afrique en 417. Quand l'évêque d'Hippone vint de si loin lui faire visite, Bonifatius traversait une crise grave : il venait de perdre sa femme et voulait abandonner la carrière des armes pour embrasser la vie monastique ; Augustin et Alypius lui représentèrent quel service il rendrait à l'Église si, demeurant dans sa charge, il continuait à la défendre contre les attaques des Barbares (Ep. 220, 3, CSEL, 57, p. 443).

\section{La « cura pastoralis " et la « tuitio episcopalis" : l'évêque dans son diocèse d'Hippone}

25 Si chargé fût-il de responsabilités dans le cadre de son Afrique, de Carthage à Caesarea, c'est à l'intérieur de son évêché et dans ses parages immédiats, dans le terroir qu'il contrôlait étroitement avec ses amis, Alypius, évêque de Thagaste et Possidius, évêque de Calama, qu'Augustin était le plus profondément immergé dans la réalité africaine : dans sa réalité religieuse en tant que pasteur, dans sa réalité sociale, et même politique, en sa qualité de chef de communauté investi d'une mission générale de protection (la tuitio episcopalis). Deux exemples suffiront à rendre sensible cette proximité locale de l'évêque avec son peuple.

26 Le diocèse dont Augustin avait reçu la charge pastorale en 395 était un des plus vastes d'Afrique. Il était en outre dans sa partie sud, vers Calama et les "Alpes numidiques ", vers Thagaste et le haut cours de la Medjerda, de relief tourmenté, avec des zones montagneuses d'accès difficile. Dans ces «marches " rurales éloignées de la ville épiscopale d'une cinquantaine de kilomètres, l'Église donatiste était par surcroît très active. Augustin comprit vite que ces dimensions géographiques et cette situation 
religieuse imposaient à l'évêque d'Hippone d'alléger sa charge pastorale en créant quelques chaires épiscopales aux confins de ce trop vaste diocèse. C'est ainsi que, principalement dans les années qui précédèrent la Conférence de 411 , des évêchés furent créés à Zattara, Thullio, Siniti, Mutugenna, Fussala. Quelques-uns de ces lieux-dits n'ont pu être identifiés, ni localisés, et c'est notamment le cas du dernier nommé, Fussala, qu'on ne peut situer sur une carte que de façon hypothétique. Les problèmes qu'Augustin avait eu à résoudre au castellum Fussalense nous sont mieux connus depuis qu'un document naguère encore inédit (la lettre $20^{*}$ : cf. CSEL, 88, p. 94-112) est venue préciser les informations contenues à ce sujet dans la lettre 209 (cf. CSEL, 57, p. 347-353) écrite par l'évêque d'Hippone au pape Célestin. Non sans mal, Augustin avait réussi à rallier à l'Église catholique les chrétiens de ce bourg de montagne situé aux confins du territoire de sa cité épiscopale et longtemps dominé par les schismatiques. L'éloignement du site (une cinquantaine de kilomètres) et les difficultés de communication l'avaient déterminé à l'ériger en évêché, à la tête duquel il plaça, faute de mieux, un des ses lecteurs, du nom d'Antoninus, qu'il avait recueilli, tout enfant, et élevé dans son monastère. Un des éléments de cette décision était qu'Antoninus parlait le punique, dont la pratique était indispensable dans bien des secteurs de la Numidie d'Hippone. Malheureusement, le jeune homme (il avait à peine plus de vingt ans) se révéla vite un évêque détestable, prévaricateur et déprédateur, qui mit en coupe réglée le petit bourg et les maigres ressources de ses fidèles. Pour le mettre hors d'état de nuire et lui faire rendre gorge, il fallut l'excommunier, le faire condamner devant un tribunal épiscopal, puis, Antoninus ayant fait appel à Rome, faire passer l'affaire en seconde instance devant une commission épiscopale composée à la demande du pape Boniface. Dans la lettre $20^{*}$ adressée à une riche aristocrate romaine, Fabiola, chez qui Antoninus avait trouvé asile, Augustin a raconté par le menu les pérégrinations de cette commission présidée par le primat de la province de Numidie, Aurelius de Macomades. On la voit se transporter de ville en ville, de fundus en uilla, le primat interrogeant les villageois en punique pour se faire préciser leurs griefs à l'égard de l'évêque Antoninus (Ep. 20*, CSEL, 88, p. 105). Chemin faisant, on voit aussi se préciser la physionomie de ces "paroisses " rurales où les propriétaires terriens (domini/dominae) ou leurs représentants (conductores) ont une influence morale et spirituelle presque aussi importante que leur poids proprement matériel. A Fussala même, gros village perché dans la montagne, avec la complicité dévoyée d'un defensor ecclesiae, d'un prêtre, d'un diacre et de quelques hommes de main, anciens soldats, Antoninus avait mis son pouvoir épiscopal au service de ses petites ambitions temporelles; avec le fruit de ses spoliations et déprédations, il s'était appliqué à faire bâtir une maison bourgeoise, tout entière faite de remplois et de matériaux d'emprunt (Ep. 20*, CSEL, 88, p. 97-98 et 110-111). A cet égard, autant qu'un très vivant document sur les chrétientés rurales de la Numidie d'Hippone, ce texte est aussi un témoignage sur une certaine misère de la culture matérielle, en dehors des grands centres urbains, en ce début du v viècle.

En face de ces misères matérielles, en face aussi des criants abus du pouvoir de potentats locaux et de graves déséquilibres sociaux, l'évêque Augustin réagissait et agissait. De ces actions de " protection épiscopale » (tuitio episcopalis), attestation nous est donnée entre autres par l'une des nouvelles lettres récemment publiées, qui montre l'évêque d'Hippone confronté au problème de la «traite » dont étaient victimes des hommes et des femmes, et même des enfants, enlevés par des bandes armées et vendus à des trafiquants d'esclaves (mangones). En ces temps de misère, les marchands d'esclaves «Galates » - leur « corporation » était en majorité originaire de Galatie, en 
Asie Mineure - avaient des «têtes de pont " sur les rivages africains et ne manquaient pas de pourvoyeurs, qui agissaient parfois par la séduction, mais le plus souvent par la violence et par le rapt. A Hippone même, une femme attirait chez elle des «marchandes de bois " de Giddaba (le Chettaba ou un autre site homonyme) et les séquestrait en attendant de pouvoir les vendre; un « colon » de l'Église (un cultivateur attaché à une propriété ecclésiastique) avait poussé la cupidité jusqu'à vendre sa femme, "la mère de ses enfants ». Mieux encore, les "Galates " avaient réussi à rassembler à Hippone, où ils étaient entassés dans des cachots en attendant leur embarquement, cent vingt malheureux, parmi lesquels quelques enfants vendus par leurs parents : un « commando » de paroissiens était parvenu à les libérer (Ep. 10*, CSEL, 88, p. 49-50).

\section{L'« africanité » de saint Augustin}

Aux yeux des contemporains, comme par exemple son rival Petilianus, évêque donatiste de Constantine, Augustin était un Africain, un Afer (cf. Contra litt. Petiliani, III, 29, CSEL, 52, p. 185 : « eo quod Afer sim »; cf. aussi ibid., III, 31, CSEL, 52, p. 186 : « quia et Afer sum »). L'évêque d'Hippone ne reniait pas ses origines, bien au contraire et, dans une lettre écrite au grammairien Maximus de Madaure, ville où il s'était ouvert enfant et adolescent à la culture classique, on le voit défendre la Punica lingua et la culture punique en termes qui révèlent, à un certain niveau, une « conscience africaine » (Ep. 17, 2, CSEL, 34, p. 41 : «neque enim usque adeo te ipsum obliuisci potuisses, ut homo Afer scribens Afris, cum simus unique in Africa constituti, Punica nomina exagitanda cogitares »). Et c'est non sans fierté qu'à deux reprises Augustin évoque, comme on fait d'une gloire nationale, l'origo africaine d'Apulée (De civ. dei, 8, 12, CSEL, 40, p. 1374 : «in utraque lingua, id est Graeca et Latina, Apuleius enim... qui nobis Afris Afer est notior»). Toutefois cette Afrique dont il se réclame, sans en renier la composante culturelle punique (même s'il ne connaissait lui-même que des bribes de l'ancienne langue de Carthage), est une Afrique profondément intégrée à la romanité et exempte de tout particularisme politique; le seul vrai particularisme à retenir chez l'évêque d'Hippone est son sentiment d'appartenir à une Église qui défendait jalousement son autonomie au sein de la catholicité et devant le siège de Rome.

On conclura sur un paradoxe. Cet Augustin qui, comme nous l'avons dit en commençant, avait, statistiquement, toutes chances d'être "berbère ", semble bien avoir ignoré les parlers indigènes de son temps. Évoquant en termes de bilinguisme les langues parlées en Afrique, il met sur le même plan la langue latine et la langue punique, c'est-à-dire, ajoute-t-il, « africaine » (Tract, in Epist. Johannis, 2, 3 : «sic honorant Christum donatistae ut dicant illum remansisse ad duas linguas, Latinam et Punicam, id est Afram »). Bien que la question soit controversée, on admet généralement, avec de bons arguments, que par Punica lingua il faut entendre, non pas le libyque, mais bien l'ancienne langue de Carthage, dont on mesure mieux maintenant quelle persistance elle a manifesté en Afrique romaine. Mais alors il faut se résigner à admettre que l'évêque d'Hippone ait ignoré les parlers proprement indigènes de son pays auxquels il fait allusion une seule fois, dans une phrase de la Cité de Dieu où seule sa méconnaissance des idiomes libyques lui permet de les réduire à l'unité : «En Afrique aussi, nous connaissons bien des peuples barbares n'ayant qu'une seule langue » (De civ. Dei, XVI, 6, 2: «Nam et in Africa barbaras gentes in una lingua plurimas nouimus»). Aussi 
bien, pour l'évêque d'Hippone, c'était la face d'ombre de son Afrique, ces Afri barbari qu'il incluait, vers la fin de sa vie, dans la montée des périls qu'il sentait venir (Ep. 220, 7, CSEL, 57, p. 436 : «Quid autem dicam de uastatione Africae, quam faciunt Afri barbari resistente nullo?»).

Pour Augustin, ces Afri barbari, qui ne parlaient ni latin ni punique, étaient essentiellement des groupes tribaux situés extra limitem, au-delà de la frontière de l'Empire vers le sud, comme les Arzuges non touchés par la romanité, ni par le christianisme.

\section{BIBLIOGRAPHIE}

MARROU H.I., Saint Augustin et l'augustinisme, Paris, Ed. du Seuil, coll. « Maîtres spirituels », s.d., 1955.

VAN DER MEER F., Saint Augustin pasteur d'âmes, 2 vol. , Paris, Et. Aug., s.d., 1959.

BROWN P.R.L., Augustine of Hippo. A Biography, Londres, Faber \& Faber, s.d., 1967.

MANDOUZE A., Saint Augustin, l'aventure de la raison et de la grâce, Paris, Et. Aug., 1968.

PERLER O. et MAIER J.L., Les voyages de Saint Augustin, Paris, Et. Aug., 1969. Les lettres de saint Augustin découvertes par J. Divjak, Communications présentées au colloque des 20 et 21 septembre 1982. Paris, Et. Aug., 1983.

INDEX

Mots-clés : Antiquité, Biographie, Christianisme 